ARTICLE

\title{
Cooperative stator assembly of bacterial flagellar motor mediated by rotation
}

Kenta I. Ito (iD ${ }^{1}$, Shuichi Nakamura ${ }^{1} \&$ Shoichi Toyabe (iD ${ }^{1 凶}$

Cooperativity has a central place in biological regulation, providing robust and highlysensitive regulation. The bacterial flagellar motor implements autonomous torque regulation based on the stator's dynamic structure; the stator units bind to and dissociate from the motor dynamically in response to environmental changes. However, the mechanism of this dynamic assembly is not fully understood. Here, we demonstrate the cooperativity in the stator assembly dynamics. The binding is slow at the stalled state, but externally forced rotation as well as driving by motor torque in either direction boosts the stator binding. Hence, once a stator unit binds, it drives the rotor and triggers the avalanche of succeeding bindings. This cooperative mechanism based on nonequilibrium allostery accords with the recently-proposed gear-type coupling between the rotor and stator.

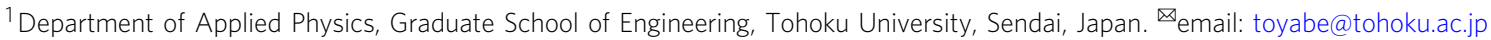


$\mathrm{M}$ otility regulation is vital and the basis of autonomy for many life forms. Swimming bacteria like Escherichia coli and Salmonella enterica have been serving as the model system of motility regulation. Since they utilize the propulsive force of the rotating flagella, the torque regulation of the flagellar motor, as well as the control of tumbling frequency and flagellar bundle formation, is essential for the motility regulation. The bacterial flagellar motor (BFM $)^{1-4}$ (Fig. 1a) is a large protein complex and can rotate at up to $1700 \mathrm{~Hz}^{5}$, reverse the rotation, and vary the torque depending on the load on the motor. A striking fact is that the BFM has multiple torque-generating stator units and regulates the torque magnitude by dynamically alternating the units between the motor and surrounding membrane pool $^{6-8}$ (Fig. 1b). This autonomous stator assembly relies on the self-regulation of each stator unit. The stator unit implements a load sensor and a load-dependent regulator ${ }^{9-12}$; the stator-unit dissociation is suppressed at high motor load for keeping large torque generation and enhanced at low load for probably reducing futile energy consumption. On the other hand, the binding rate is thought to be not significantly dependent on the load ${ }^{11}$. The ion flux through the ion channel of the stator unit is required for the stator-unit binding ${ }^{13-15}$. However, it is not clear how the ion flux is involved in the binding process.

Cooperativity is central to biological regulation ${ }^{16}$. A simple allosteric mechanism provided by the mutual dependence of the ligand-binding sites exhibits a steep nonlinear response, enabling robust and highly sensitive regulation and signaling ${ }^{17}$. It would be natural to suppose that the BFM's torque regulation is highly optimized.

We exploit the dynamic load control by the electrorotation ${ }^{18,19}$ to study the stator remodeling process ${ }^{12,20}$. The electrorotation uses a rotating high-frequency electric field in the order of $\mathrm{MHz}$ to apply external torque on microscopic dielectric objects such as the bacterial cell body. Hence, we can apply torque on the motor by tethering the flagellum to a glass surface. Since the torque magnitude is proportional to the electric field's squared amplitude $V_{0}^{2}$, we can modulate the torque dynamically.

In this work, we demonstrate the cooperative stator assembly mediated by rotation. The stator binding is slow at the stalled state, but the rotation in either direction boosts the stator binding. Since the binding of the first stator unit causes the rotation, there is a cooperative feedback loop of the stator assembly via rotation. a

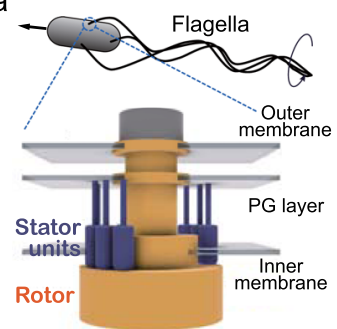

b

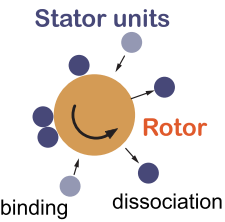

Fig. 1 Bacterial flagellar motor. a The bacterial flagellar motor is a supramolecular complex with a diameter of about $45 \mathrm{~nm}$ spanning the membrane ${ }^{1-4}$. The motor consists of a rotor (yellow) and multiple torquegenerating stator units (blue). The stator units have ion channels and generate torque at the interface with the rotor by exploiting the free energy change liberated by the ion currents through the channels. The stator units interact with the rotor at its bottom part and are anchored to the rigid peptidoglycan (PG) layer with its top part ${ }^{25-27}$. b The stator units bind to and dissociate from the motor.

\section{Results}

Stator remodeling. Each experimental cycle consists of the initial, assisting, and remodeling phases (Fig. 2a). In the initial phase, we observed a steady rotation for $10 \mathrm{~s}$ in the absence of an external load. The number of the bound stator units, $N$, was estimated to be approximately ten in this phase by the step analysis below. This relatively large value of $N$ is due to the high viscous load on the tethered cell and is consistent with the previous results ${ }^{20}$.

In the assisting phase, we assisted the rotation strongly for $10 \mathrm{~s}$ by the electrorotation for reducing the load on the motor. The

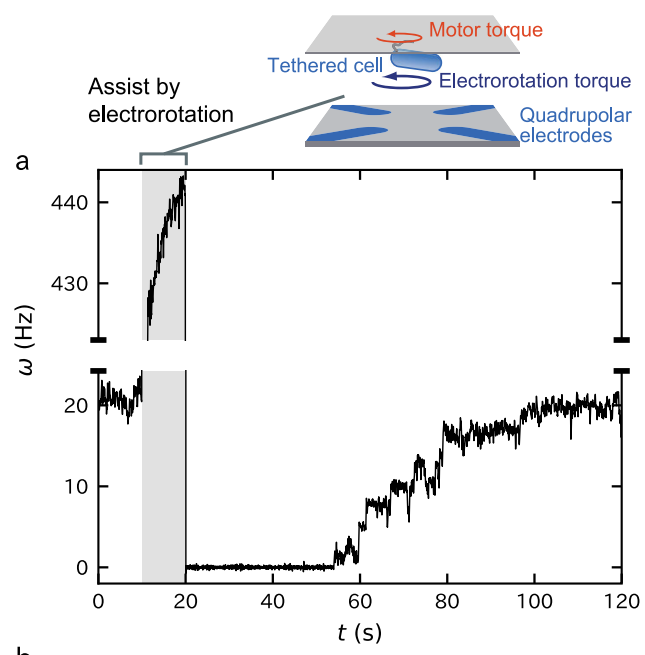

b
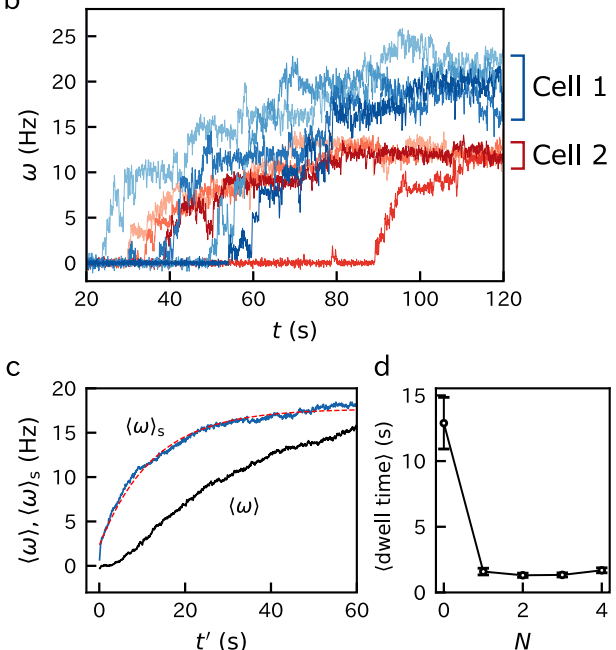

Fig. 2 Rotation traces. a A typical rotation trace of the remodeling process. After the initial 10-s phase, a strong assisting torque was applied for $10 \mathrm{~s}$ for releasing the stator units from the motor by the electrorotation method $^{20} . \omega>0$ corresponds to the counter-clockwise (CCW) direction. b Consecutive traces of two motors (blue and red) are superposed. Different colors correspond to different runs. See Supplementary Fig. 2 for the separate plots of these cells for the comparison. c Ensemble average speeds of remodeling traces, plotted against time after electrorotation stopped $\left(t^{\prime}=t-20 \mathrm{~s},\langle\omega\rangle\right.$, black) and against time after the first stator is stably incorporated $\left(t^{\prime}=t-t_{1},\langle\omega\rangle_{s}\right.$, blue). Forty traces were averaged, see text for the definition of $t_{1}$. Forty traces were averaged. For $\langle\omega\rangle$, the time origin was reset to $t^{\prime}=0 .\langle\omega\rangle_{\mathrm{s}}$ was fitted by $\langle\omega\rangle_{\mathrm{s}}=\omega_{\mathrm{ss}}\left(1-e^{-k t^{\prime}}\right)+\omega_{1} e^{-k t^{\prime}}$ with the fitting parameters of $k=0.080 \mathrm{~s}^{-1}$ and $\omega_{\mathrm{ss}}=18 \mathrm{~s}^{-1}$ (red dashed curve). $\omega_{1}$ was approximated by $\omega_{\max } / 10$. d The mean dwell time at each $N$ obtained by the step analysis of the above 40 traces (Supplementary

Fig. 3). Data are presented as mean values \pm standard errors. The number of dwells are $63,87,90,104$, and 135 for $N=0, \ldots, 4$, respectively. 
rotation speed was accelerated to about $300-600 \mathrm{~Hz}$ depending on the body dimension and cellular dielectric property.

In the remodeling phase, the assisting torque was turned off. The rotation rate vanished in the most traces (about $80 \%$ of the observed traces). The motor stalling indicates that all the bound stator units are released under the low-load condition ${ }^{12,20}$. Some motors continued rotating while their rotation rate decreased much compared to the initial phase. This may be due to the insufficient magnitude and duration of the assisting torque.

The rotation rate recovered to the initial steady level with successive jumps, indicating the stepwise stator-unit bindings. The duration of each cycle was $120 \mathrm{~s}$. We repeated multiple cycles for the same motor.

Slow binding of the first stator unit. We found strong stochasticity in the traces of the rotation rate $\omega$, especially at small $\omega$ (Fig. 2b). The duration of the zero-speed state was typically long compared to the duration of the other states. That is, the binding rate of the first stator unit is small. However, once a stator unit binds, it triggers the bindings of succeeding stator units.

The slow binding at $N=0$ is highlighted when we compared the ensemble average of the traces plotted against time after electrorotation stopped $\left(t^{\prime}=t-20 \mathrm{~s},\langle\omega\rangle\right)$ and that plotted against time after the first stator is stably incorporated $\left(t^{\prime}=t-t_{1},\langle\omega\rangle_{s}\right)$. We located the timing of the stable binding $t_{1}$ as $\omega$ exceeded $\omega_{c} \equiv \omega_{\max } / 20$ (see Supplementary Fig. 1 for the distribution of $\left.t_{1}\right) \cdot \omega_{\max }$ is the maximum rotation rate except for the duration where external torque is induced. Since approximately ten stator units bind at the steady-state, $\omega_{c}$ corresponds to half of the rotation rate caused by a single stator unit. When the traces intersected $\omega_{c}$ multiple times, we used the last one. We found that $\langle\omega\rangle_{\mathrm{s}}$ deviated significantly from $\langle\omega\rangle$. $\langle\omega\rangle$ showed a downward convexity at small $t^{\prime}$ in contrast to the upward convexity of $\langle\omega\rangle_{s}$. This characteristic is expected to be caused by the long duration of $N=0$ state.

A theoretical curve, $\langle\omega\rangle_{\mathrm{s}}=\omega_{\mathrm{ss}}\left(1-e^{-k t^{\prime}}\right)+\omega_{1} e^{-k t^{\prime}}$, fitted $\langle\omega\rangle_{\mathrm{s}}$ well with the fitting parameters of the steady-state rotation rate $\omega_{\mathrm{ss}}=18 \mathrm{~s}^{-1}$ and the rate constant $k=0.080 \mathrm{~s}^{-1}$ (Fig. 2c). $\omega_{1}$ is the rotation rate caused by a single stator unit and was approximated by $\omega_{\max } / 10$. This curve is the solution of the random packing model ${ }^{11}$ given by $d\langle\omega\rangle_{s} / d t^{\prime}=k\left(\omega_{s s}-\langle\omega\rangle_{s}\right)$ under the initial condition of $\langle\omega\rangle_{s}(0)=\omega_{1} . k$ is the sum of the binding rate at a binding site $k_{+}$and the dissociation rate of a bound stator $k_{-}$. The successful fitting may validate the model assumption for $N \geq 1 ; k_{ \pm}$do not have significant dependence on $N$. See references ${ }^{11,12}$ for detailed analysis.

The step analysis of the remodeling traces (Supplementary Fig. 3) supported the long pausing at $N=0$ compared to those at $N \geq 1$ (Fig. 2d). This implies that $N=0$ is a metastable state.

Additional experiments were done to eliminate the possibility that the slow binding at $N=0$ is caused by an experimental artifact. The possible concerns include the cell-surface interaction such as sticking to the surface and the mechanical or thermal damage of the cells caused by the electrorotation. As the test, we observed remodeling traces without experimental measures that were designed to minimize these possible artifacts. We used cells without a shearing treatment and blocking agent with reduced concentration $(50 \mathrm{mg} / \mathrm{ml})$, which expectedly caused more frequent cell sticking to the surface. We also used the observation buffer containing high ion strength $(10 \mathrm{mM}$ MOPS and $10 \mathrm{mM}$ $\mathrm{KCl}$ ), which increased the temperature three times as much as the present condition during the assisting phase (Supplementary Fig. 4). Nonetheless, we obtained quantitatively similar remodeling traces and dwell time (Supplementary Fig. 5). Furthermore, when we divided the data into two groups according to the a

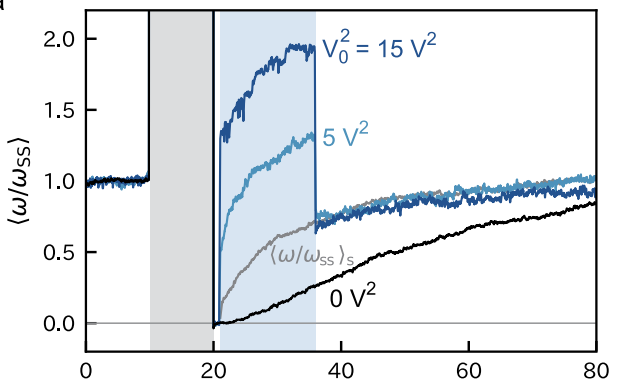

b
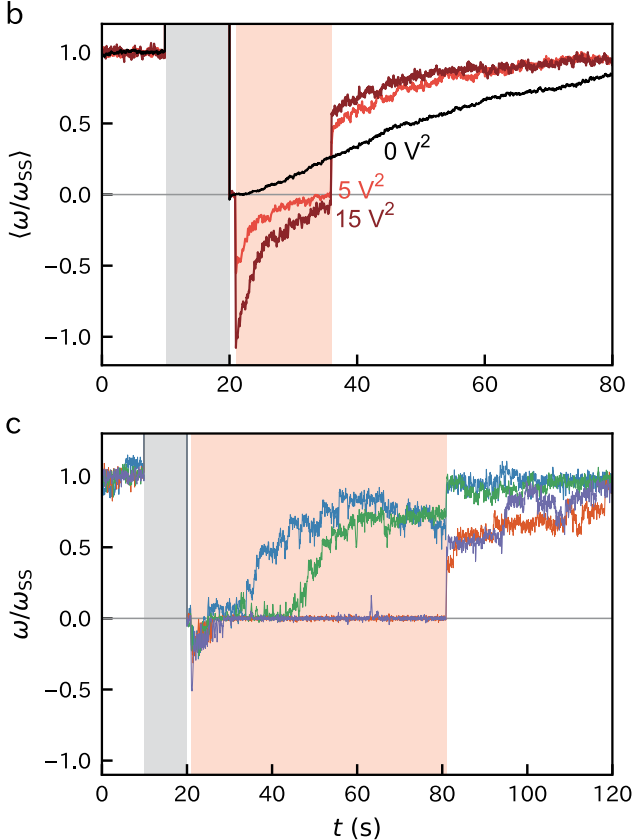

Fig. 3 Remodeling under external torque. Ensemble averages of the remodeling traces under constant external torque in the assisting direction (a) and hindering direction (b) with different $V_{0}^{2}$. The torque was induced for $15 \mathrm{~s}(\mathbf{a}, \mathbf{b})$ or $60 \mathrm{~s}(\mathbf{c})$ starting at $21 \mathrm{~s}$. The rotation rate $\omega$ was first normalized by the steady rotation rate $\omega_{\text {ss }}$ calculated as the mean $\omega$ during the initial phase and then averaged. The black and gray curves are the calibrated curves of $\langle\omega\rangle$ and $\langle\omega\rangle_{\mathrm{s}}$ of Fig. 2c. For $\langle\omega\rangle_{\mathrm{s}}$ the time origin was shifted to $t=21 \mathrm{~s}$. See also Supplementary Fig. 6 for the combined plots of $\mathbf{a}$ and $\mathbf{b}$ for comparison. $\mathbf{c}$, The remodeling process with extended torque induction in the hindering direction. Different colors correspond to different runs. $V_{0}^{2}=3$ (blue), 5 (green, orange), and $10 \mathrm{~V}^{2}$ (purple).

rotation rate during the assisting phase, we did not find a significant difference of the $N=0$ duration between them $(12.3 \pm$ $3.0 \mathrm{~s}$ and $13.4 \pm 2.6 \mathrm{~s}$ for the faster and slower groups, respectively (mean \pm standard errors)). If the electrorotation made damage to the motor, the damage magnitude would increase with the forced rotation rate. These results support that the slow binding at $N=0$ is the motor characteristic. This will be further verified below; the binding at $N=0$ is strongly enhanced by forced rotation.

Rotation enhances binding. For elucidating the mechanism of the slow binding at $N=0$, we investigated the remodeling process under forced rotation (Fig. 3). We calibrated the rotation rate by dividing by the steady-state rotation rate during the initial phase $\omega_{\text {ss. }}$. The assisting phase was followed by a 1-s duration without external torque for verifying that all the stator units are released. We analyzed only the trajectories with vanished rotation rates. Then, a constant external torque was applied for $15 \mathrm{~s}$. Accordingly, a steep change in $\omega$ was observed at the start and end of the 
torque induction. These jumps had similar magnitudes and opposite signs as expected.

Under the forced rotation in either direction, the remodeling proceeded quickly with upward convexity (Fig. 3a, b). As well, at the timing when assisting torque was turned off, the values of $\left\langle\omega / \omega_{\text {ss }}\right\rangle$ under forced rotation were larger than that without forced rotation (Fig. 3a). That is more stator units bound with the forced rotation, suggesting that the rotation enhances the binding. The curves under different external torque, as well as $\left\langle\omega / \omega_{\text {ss }}\right\rangle_{\text {s }}$ were similar except for the bias during the torque induction and coincided well after the torque was turned off. This indicates that the binding dynamics do not significantly depend on the magnitude of the external driving.

Interestingly, the remodeling dynamics under the assisting and hindering torque were asymmetric. When assisted, the remodeling continued until $\omega$ recovers to the level of the initial phase (Fig. 3a and Supplementary Fig. 7). On the other hand, when hindered, the remodeling started quickly as well but stalled when $\omega$ reached zero (Figs. 3b, c, and Supplementary Fig. 7). The stall typically continued until the removal of the induced torque, and then the remodeling restarted quickly. This characteristic did not change qualitatively with a different magnitude of hindering torque. Further remodeling during the stall was occasionally observed when we extended the duration of torque induction (Fig. 3c). These results suggest that the binding is slow even for $N \neq 0$ if $|\omega|$ vanishes and is enhanced by the rotation in either direction.

During the stall under hindering, the motor torque $T_{\mathrm{m}}$ and electrorotation torque $T_{\text {ex }}$ should be balanced. However, this is not expected considering the discrete nature of $T_{\mathrm{m}}$. It is expected that $T_{\mathrm{m}}$ takes discrete values proportional to $N$ under the present high load condition of the tethered cell assay. That is, the perfect balance between $T_{\mathrm{m}}$ and $T_{\mathrm{ex}}$ is not expected. Nevertheless, the remodeling under hindering torque experienced the stall in most trajectories. This is probably due to the pinning of the cell caused by, for example, tiny obstacles on the glass surface.

We observed that when the cells are forcedly rotated at a slow rate of approx. $1 \mathrm{~Hz}$, the further binding did not proceed quickly (Supplementary Fig. 7a). The binding enhancement by rotation may have a threshold for $\omega$. However, we had difficulty in determining the threshold value since the slow rotations suffered from the cell-surface interaction and stopped occasionally.

Quantitative evaluation of binding enhancement under forced rotation. We evaluated the binding enhancement under forced rotation. We exploited pulse-wise external torque instead of the constant torque (Fig. $4 \mathrm{a}, \mathrm{b}$ ). This is because the binding may occur very soon after the torque induction. If this is the case, it is difficult to distinguish the increase in $\omega$ by the torque induction and that by the stator-unit binding.

We repeated short torque induction (ON) lasting for $\tau=0.5 \mathrm{~s}$ with a one-second break (OFF) during the remodeling phase. We assumed that the first stator unit bound during an $\mathrm{ON}$ when the mean of $\omega$ in the succeeding OFF exceeded $\omega_{c}$. We counted the number of binding events $(N=0 \rightarrow 1), n_{+}$, and non-binding events $(N=0 \rightarrow 0), n_{0}$, at $N=0$. These numbers were accumulated for multiple traces. We used 396 traces of 122 cells in total. The fraction $r=n_{+} /\left(n_{+}+n_{0}\right)$ quantifies how the forced rotation enhances the binding.

The forced rotation in either direction significantly enhanced the binding (Fig. 4c). $r$ depended on the rotational direction and was larger for the clockwise $(\mathrm{CW})$ rotation than for the counterclockwise (CCW) rotation. A significant dependence of $r$ on $\omega$ was not observed. Specifically, we could not resolve the gradual increase in $r$ at small $\omega$. The plots with varied bin widths indicated that the threshold value of $|\omega|$ for the binding enhancement was estimated to be less than approx. $3 \mathrm{~Hz}$ (Supplementary Fig. 8).

Note that the binding rate at a single binding site is approximated by $\tilde{b}=b / N_{\max }$ with $b=-\tau^{-1} \ln (1-r)$. Here, the bound stator unit is supposed not to dissociate during the same ON. The probability that an event obeying a Poissonian process with the rate $b$ takes place within $\tau$ is $r=1-\exp (-b \tau)$, which leads to the above relation. The average of $b$ in the CCW rotation was $1.3 \pm 0.1 \mathrm{~s}^{-1}$ (Supplementary Fig. 8), which yields $\tilde{b} \sim 0.09 \mathrm{~s}^{-1}$ assuming $N_{\max }=14$. On the other hand, the fitting in Fig. 2c provided $k=k_{+}+k_{-}=0.080 \mathrm{~s}^{-1}$, which corresponds to the rate constant under the CCW rotation driven internally by the stator units. For the tethered cells, $k \simeq k_{+}$is expected since $k_{-}$ is negligible at high load ${ }^{11}$. Hence, the similarity of $k$ and $\tilde{b}$ implies that, at least in the CCW rotation, the binding rate is solely determined by the rotation rate independent of whether the rotor is externally rotated or internally rotated by the stator units.

\section{Discussion}

We found that the binding is significantly enhanced by the rotation in either direction with $|\omega|$ exceeding a small threshold. The CW rotation was more effective than the CCW rotation. The binding of a stator unit induces the rotation and, therefore, triggers the succeeding stator-unit bindings like an avalanche. Thus, the nonequilibrium allostery via rotation mediates the cooperativity of the stator assembly. The evaluation of the threshold values and the binding and dissociation rate under forced rotation remains for future studies. The ion flux is required for the stator assembly ${ }^{13-15}$. Without the ion flux, the stator units do not generate torque and rotate the rotor. This is consistent with our result.

Similar experiments were previously done for examining the dissociation during the assisting phase ${ }^{12}$. They also analyzed the remodeling process in the absence of external torque. However, they studied the long-timescale dynamics of approx. $200 \mathrm{~s}$ and did not focus on the short timescale, where the slow binding at $N=0$ is effective. In such a long timescale, we did not see a significant difference between $\langle\omega\rangle$ in Fig. $2 c$ and their curve despite that they used the BFM of E. coli (Supplementary Fig. 9).

It was previously shown that the binding rate does not depend on the load at intermediate $N^{11}$, while the dissociation rate has a significant dependence on the load ${ }^{11,12}$. We showed that the binding is enhanced in either rotational direction despite that the load on the stator is different between them; small or negative with the assisting torque and large with the hindering torque. This supports that the binding is regulated not by the load on the previously bound stator units even at small $N$ but by the rotation rate. It is natural that the stator unit that is not yet incorporated does not feel the load. A question is how the stator unit can sense the rotation.

The structure of the stator unit implies that the stator unit itself is a rotating motor. The $\operatorname{Mot}_{5}$ ring may revolve around the $\mathrm{MotB}_{2}$ axis and rotate the rotor via a gear-type coupling ${ }^{21-23}$ (Fig. 5). Such coupling may explain our finding that the rotor rotation accelerates the binding of the stator unit as follows.

A reasonable hypothesis for the binding process is that the stator unit diffusing in the membrane pool collides with the motor, binds partially to the rotor, and will be activated. Then, the stator unit starts torque generation. The stator-unit binding involves the interaction between the MotA and $\operatorname{rotor}^{24}$ and the binding of MotB's C-terminal to the peptidoglycan (PG) layer as an anchor $^{25-27}$.

Accordingly, there are at least two possibilities for the rotationsensing mechanism. First, the stator unit may sense the rotation 

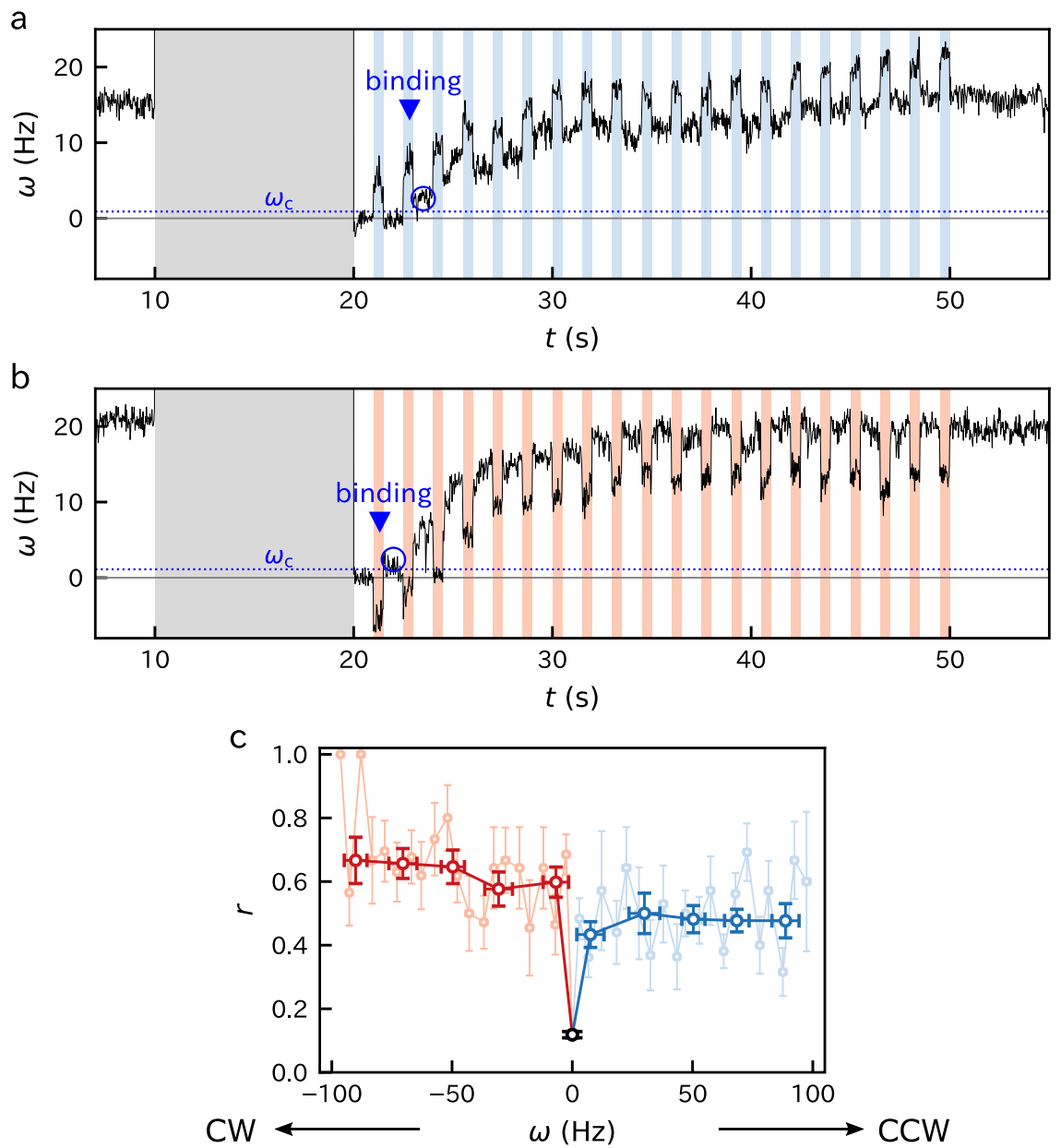

Fig. 4 Evaluation of rotation-dependent binding rate using pulse-wise forced rotation. The alternation of 0.5-s ON (shaded by blue or red) and 1-s OFF of the external torque was induced in assisting direction (a) or hindering direction (b). When $\omega$ exceeded $\omega_{\mathrm{c}} \equiv \omega_{\max } / 20$, we assumed that the first stator unit bound. c, Binding rate as a function of the forced rotation rate averaged in bins with the width of $20 \mathrm{~Hz}$ (dark) superposed on that with $5-\mathrm{Hz}$ bins (light). We used 396 traces of 122 cells in total. See Supplementary Fig. 8 for details. Data are presented as mean values \pm standard errors.

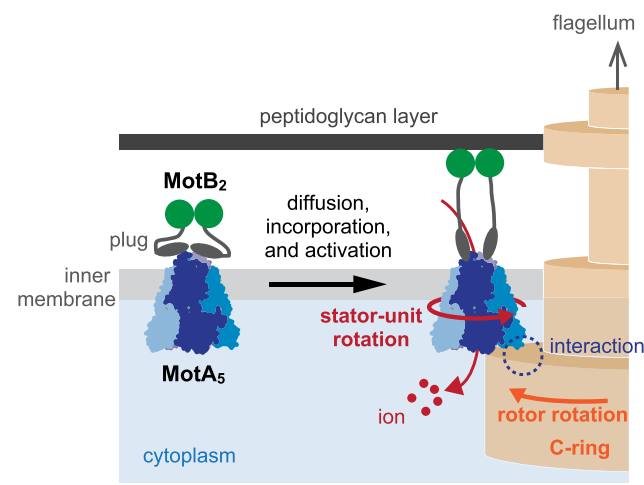

Fig. 5 The binding process of the stator unit to the rotor. The freelydiffusion stator unit collides with the rotor. The electrostatic interaction with the C-ring induces a conformational change of the stator unit, which allows the ion flow and, hence, the torque generation.

via the gear-type coupling between the MotA and rotor. The rotor rotation may rotate the stator unit via the gear and induce the stator unit's conformational change necessary for the activation. It is natural to assume that such rotation-mediated activation can be differently induced by the $\mathrm{CW}$ and CCW rotation (Fig. 4c).

Second, the stator unit may sense the rotation via the anchoring of the stretched MotB's C-terminal to the PG layer.
Assume that the MotB is already bound to the PG layer before the activation. Then, the rotor rotation may apply torque on the stator unit along the rotor periphery and pull the $\operatorname{Mot}^{11}$. Such a pulling force may induce the conformational change of the stator unit and activate the stator unit. The magnitude of the pulling force would be determined by the balance of torque applied to the stator unit. Therefore, if this partially bound stator unit generates torque, the pulling force would depend on the rotation direction of the rotor. This may explain the CW-CCW asymmetry of the activation rate. We need further research to conclude.

The metastability of the stalled state may be beneficial for bacteria by ensuring that the rotor does not rotate during the motor assembly and that futile binding more than necessary does not occur when stacked in biofilm. More studies would clarify the function served by the binding enhancement by rotation.

An intriguing question related to the function is whether a rotational fluctuation enhances the binding or unidirectional rotation is necessary. The motor under the swimming condition is expected to have larger rotational fluctuations at a stall than those of the tethered cell. The determination of the threshold rotation rate for the binding enhancement may provide a hint for answering this question.

We evaluated the stator-assembly dynamics during the remodeling process based on the dynamical load control provided by the electrorotation. The results highlighted the cooperativity in the stator assembly. The stalled state $(\omega=0)$ is meta-stable, and 
the binding of the stator unit to the stalled rotor is slow. The rotation driven by an external torque, as well as motor torque in either direction enhances the stator binding. The binding of the first stator unit causes the rotation and hence enhances further bindings of stator units. The elucidation of the molecular mechanism behind this cooperative feedback remains for future studies.

\section{Methods}

Cell preparation. Salmonella strain YSC2123, which lacks motA, motB, cheY, fimA, and fliC (204-292), was transformed with a plasmid encoding wild-type motA/ mot $B^{28}$ (referred to as a wild-type). This strain has a "sticky" filament with a hydrophobic surface beneficial for the tethered cell assay. Cells were grown in Lbroth containing $100 \mu \mathrm{g} / \mathrm{ml}$ ampicillin and $0.2 \%$ arabinose (for the expression of the MotA and MotB) for at least $10 \mathrm{~h}$ at $30^{\circ} \mathrm{C}$ with shaking. The buffer was replaced with the observation buffer (5 mM MOPS(3-Morpholinopropanesulfonic acid) and $5 \mathrm{mM} \mathrm{KCl}$ adjusted to $\mathrm{pH} 8.3$ with $\mathrm{KOH}$ ). We partially sheared the flagella filaments by passing the bacterial solution through $25 \mathrm{G}$ needle 70 times.

Microscopy. We observed the rotation of a tethered cell at room temperature $\left(24^{\circ} \mathrm{C}\right)$ on a phase-contrast upright microscope (Olympus BX51WI, Japan) with a $\times 60$ objective lens (Olympus, $\mathrm{NA}=1.42$ ), at $4000 \mathrm{~Hz}$ using a high-speed CMOS camera (Basler, Germany), high-intensity LED $(623 \mathrm{~nm}, 4.8 \mathrm{~W}$, Thorlabs, NJ) for illumination, and a laboratory-made capturing software developed on LabVIEW (National Instruments, TX). The angular position of the cellular body was analyzed by an algorithm based on a principal component analysis of the cell image. We used $250 \mathrm{mg} / \mathrm{ml}$ Perfect Block (MobiTec, Germany) as the blocking agent to suppress the interaction between the cell body and the glass surface. The chamber height was about $20 \mu \mathrm{m}$

Electrorotation. A $10-\mathrm{MHz}$ sinusoidal voltage with a phase shift of $\pi / 2$ was induced on the four electrodes patterned on the bottom glass surface (Fig. 1a). The distance between the electrodes is $47 \mu \mathrm{m}$. The signal was generated by a function generator (nf, Japan) controlled by PC and divided by $180^{\circ}$ phase distributors (Thamway, Japan). They were amplified by four amplifiers (Analog Devices, MA) and loaded on the electrodes. This generates an electric field rotating at $10 \mathrm{MHz}$ in the center of the electrodes and induces a dipole moment rotating at $10 \mathrm{MHz}$ on the cell body. Since there is a phase delay between the electric field and the dipole moment, the cell body is subjected to a constant torque. The torque magnitude is proportional to the square of the voltages' amplitude $V_{0}$. We modulated $V_{0}$ by a signal generated by the multifunction board (National Instruments) equipped on PC. The camera and amplitude signal were synchronized at a time difference of less than one microsecond. We applied an external torque with a time-dependent magnitude, as indicated in the main text, with superposed by a $1000-\mathrm{Hz}$ smal sinusoidal torque for the possible torque calibrations ${ }^{20,29}$ although we did not calibrate torque in this work.

The temperature under electrorotation was measured using a pair of temperature-dependent fluorescent dyes, Rhodamine B and Rhodamine 101, with different temperature dependence ${ }^{30}$ (Supplementary Methods 1 ). The ratio of the fluorescence of these dyes provides the temperature. The estimated temperature during electrorotation was $28.1^{\circ} \mathrm{C}$ for $V_{0}^{2}=240 \mathrm{~V}^{2}$, which is the typical value used during the assisting phase, at the room temperature of $24^{\circ} \mathrm{C}$ (Supplementary Fig. 4). The temperature relaxed to room temperature soon within a second after the electrorotation was turned off.

Step analysis. We estimated the dwell time at small $N$ during the remodeling process by a step analysis algorithm developed recently ${ }^{10,12}$ (Fig. 2d). The trajectories are typically noisy. We analyzed 40 remodeling traces (19 cells) with seemingly clear steps. Approximately the same number of erroneous traces was not used.

The algorithm tries to find the large velocity jump in the traces by comparing the mean velocities of adjacent segments of the traces. The core part of the algorithm is briefly summarized in the following. The traces were first averaged with moving windows with the length of 500 frames and 100 -frame shift. Then, the traces were divided into small segments with a length of 5 points. The adjacent segments were merged when the mean velocities were smaller than a threshold value, $\omega_{\max } / 15$. Here, $\omega_{\max }$ is the maximum of the moving-averaged $\omega$ during the initial phase and also the last part of the remodeling phase $(t \geq 50 \mathrm{~s})$. This procedure was iterated until adjacent segments could no longer be merged. Then, the segment boundaries were adjusted so that the $\chi^{2}$ for the two segments is minimized. If this adjustment generates a segment shorter than 5 points, the segment was merged to the adjacent segment.

Binding rate. Let $t_{i}$ be the starting time of the $i$-th OFF state. We assumed that the stator unit bound in the $i$-th ON state if $\omega_{i+1}^{\text {off }}>\omega_{\mathrm{c}}=\omega_{\max } / 20$. Here, $\omega_{i}^{\text {off }}$ is the mean rotation rate during $t_{i}+0.05 \mathrm{~s} \leq t<t_{i}+0.15 \mathrm{~s}$. This limitation avoids including the relaxation right after the torque switching and also the possible binding during the OFF state. Since approximately 10 stator units are thought to be bound at maximum under the present condition, $\omega_{\mathrm{c}}$ corresponds to half of the rotation rate caused by a single stator unit.

When the binding takes places in the $i$-th ON state, the corresponding rotation rate is determined as the mean rotation rate during $s_{i}+0.05 \mathrm{~s} \leq t \leq s_{i}+0.15 \mathrm{~s}$. Here, $s_{i}$ is the starting time of the $i$-th ON state.

\section{Data availability}

Source data are provided with this paper. The raw rotational traces that support the findings of this study are available in figshare with the identifier https://doi.org/10.6084/ m9.figshare. $14371232^{31}$. Source data are provided with this paper.

\section{Code availability}

The computer codes used for this study are available from the corresponding author upon reasonable request.

Received: 27 April 2020; Accepted: 28 April 2021; Published online: 28 May 2021

\section{References}

1. Berg, H. C. The rotary motor of bacterial flagella. Annu. Rev. Biochem. 72, 19-54 (2003).

2. Berry, R. M. \& Armitage, J. P. The bacterial flagella motor. Adv. Microb. Physiol. 41, 291-337 (1999).

3. Sowa, Y. \& Berry, R. M. Bacterial flagellar motor. Q. Rev. Biophys. 41, 103-132 (2008).

4. Nakamura, S. \& Minamino, T. Flagella-driven motility of bacteria Biomolecules 9, 279 (2019).

5. Magariyama, Y. et al. Very fast flagellar rotation. Nature 371, 752-752 (1994)

6. Block, S. M. \& Berg, H. C. Successive incorporation of force-generating units in the bacterial rotary motor. Nature 309, 470-2 (1984).

7. Ryu, W. S., Berry, R. M. \& Berg, H. C. Torque-generating units of the flagellar motor of Escherichia coli have a high duty ratio. Nature 403, 444 (2000).

8. Leake, M. C. et al. Stoichiometry and turnover in single, functioning membrane protein complexes. Nature 443, 355 (2006).

9. Tipping, M. J., Delalez, N. J., Lim, R., Berry, R. M. \& Armitage, J. P. Loaddependent assembly of the bacterial flagellar motor. mBio 4, 00551-13 (2013).

10. Lele, P. P., Hosu, B. G. \& Berg, H. C. Dynamics of mechanosensing in the bacterial flagellar motor. PNAS 110, 11839-44 (2013).

11. Nord, A. L. et al. Catch bond drives stator mechanosensitivity in the bacterial flagellar motor. PNAS 114, 12952-12957 (2017).

12. Wadhwa, N., Phillips, R. \& Berg, H. C. Torque-dependent remodeling of the bacterial flagellar motor. PNAS 116, 201904577 (2019).

13. Fukuoka, H., Wada, T., Kojima, S., Ishijima, A. \& Homma, M. Sodiumdependent dynamic assembly of membrane complexes in sodium-driven flagellar motors. Mol. Microbiol. 71, 825-835 (2009).

14. Tipping, M. J., Steel, B. C., Delalez, N. J., Berry, R. M. \& Armitage, J. P. Quantification of flagellar motor stator dynamics through in vivo protonmotive force control. Mol. Microbiol. 87, 338-347 (2013).

15. Sowa, Y., Homma, M., Ishijima, A. \& Berry, R. M. Hybrid-fuel bacterial flagellar motors in Escherichia coli. PNAS 111, 3436-3441 (2014).

16. Williamson, J. R. Cooperativity in macromolecular assembly. Nat. Chem. Biol. 4, 458-465 (2008).

17. Pauling, L. The oxygen equilibrium of hemoglobin and its structural interpretation. PNAS 21, 186-191 (1935).

18. Washizu, M. et al. Dielectrophoretic measurement of bacterial motor characteristics. IEEE Trans. Ind. Appl. 29, 286 (1993).

19. Berg, H. C. \& Turner, L. Torque generated by the flagellar motor of escherichia coil. Biophys. J. 65, 2201-2216 (1993).

20. Sato, K., Nakamura, S., Kudo, S. \& Toyabe, S. Evaluation of the duty ratio of the bacterial flagellar motor by dynamic load control. Biophys. J. 116, 1952-1959 (2019).

21. Santiveri, M. et al. Structure and function of stator units of the bacterial flagellar motor. Cell 183, 244-257.e16 (2020).

22. Deme, J. C. et al. Structures of the stator complex that drives rotation of the bacterial flagellum. Nat. Microbiol. 5, 1553-1564 (2020).

23. Chang, Y. et al. Molecular mechanism for rotational switching of the bacterial flagellar motor. Nat. Str. Mol. Biol. 27, 1041-1047 (2020).

24. Morimoto, Y. V., Nakamura, S., Kami-ike, N., Namba, K. \& Minamino, T. Charged residues in the cytoplasmic loop of MotA are required for stator assembly into the bacterial flagellar motor. Mol. Microbiol. 78, 1117-1129 (2010).

25. Kojima, S. et al. Stator assembly and activation mechanism of the flagellar motor by the periplasmic region of MotB. Mol. Microbiol. 73, 710-718 (2009). 
26. Terahara, N. et al. $\mathrm{Na}^{+}$-induced structural transition of MotPS for stator assembly of the Bacillus flagellar motor. Sci. Adv. 3, eaao4119 (2017).

27. Kojima, S. et al. The helix rearrangement in the periplasmic domain of the flagellar stator b subunit activates peptidoglycan binding and ion influx. Structure 26, 590-598.e5 (2018).

28. Morimoto, Y. V., Che, Y. S., Minamino, T. \& Namba, K. Proton-conductivity asssay of plugged and unplugged MotA/B proton channel by cytoplasminc pHluorin expressed in Salmonella. FEBS Lett. 584, 1268-72 (2010).

29. Toyabe, S., Watanabe-Nakayama, T., Okamoto, T., Kudo, S. \& Muneyuki, E. Thermodynamic efficiency and mechanochemical coupling of $F_{1}$-ATPase. PNAS 108, 17951-17956 (2011).

30. Sakamoto, Y. \& Toyabe, S. Assembly of a functional and responsive microstructure by heat bonding of DNA-grafted colloidal brick. Sci. Rep. 7, 1-6 (2017).

31. Ito, K., Nakamura, S. \& Toyabe, S. Dataset for "Cooperative stator assembly of bacterial flagellar motor mediated by rotation”. figshare https://doi.org/ 10.6084/m9.figshare.14371232 (2021)

\section{Acknowledgements}

We thank Yoshiyuki Sowa, Yohei Nakayama, and Kazuo Sasaki for helpful discussions. This work was supported by JSPS KAKENHI (16H00791 and 18H05427).

\section{Author contributions}

K.I., S.N., and S.T. designed research and wrote the paper. K.I. performed experiments. K.I. and S.T. contributed analytic tools and analyzed data.

\section{Competing interests}

The authors declare no competing interests.

\section{Additional information}

Supplementary information The online version contains supplementary material available at https://doi.org/10.1038/s41467-021-23516-y.

Correspondence and requests for materials should be addressed to S.T.

Peer review information Nature Communications thanks Richard Berry and the other, anonymous, reviewer(s) for their contribution to the peer review of this work.

Reprints and permission information is available at http://www.nature.com/reprints

Publisher's note Springer Nature remains neutral with regard to jurisdictional claims in published maps and institutional affiliations.

(c) (i) Open Access This article is licensed under a Creative Commons Attribution 4.0 International License, which permits use, sharing, adaptation, distribution and reproduction in any medium or format, as long as you give appropriate credit to the original author(s) and the source, provide a link to the Creative Commons license, and indicate if changes were made. The images or other third party material in this article are included in the article's Creative Commons license, unless indicated otherwise in a credit line to the material. If material is not included in the article's Creative Commons license and your intended use is not permitted by statutory regulation or exceeds the permitted use, you will need to obtain permission directly from the copyright holder. To view a copy of this license, visit http://creativecommons.org/ licenses/by/4.0/.

(C) The Author(s) 2021 